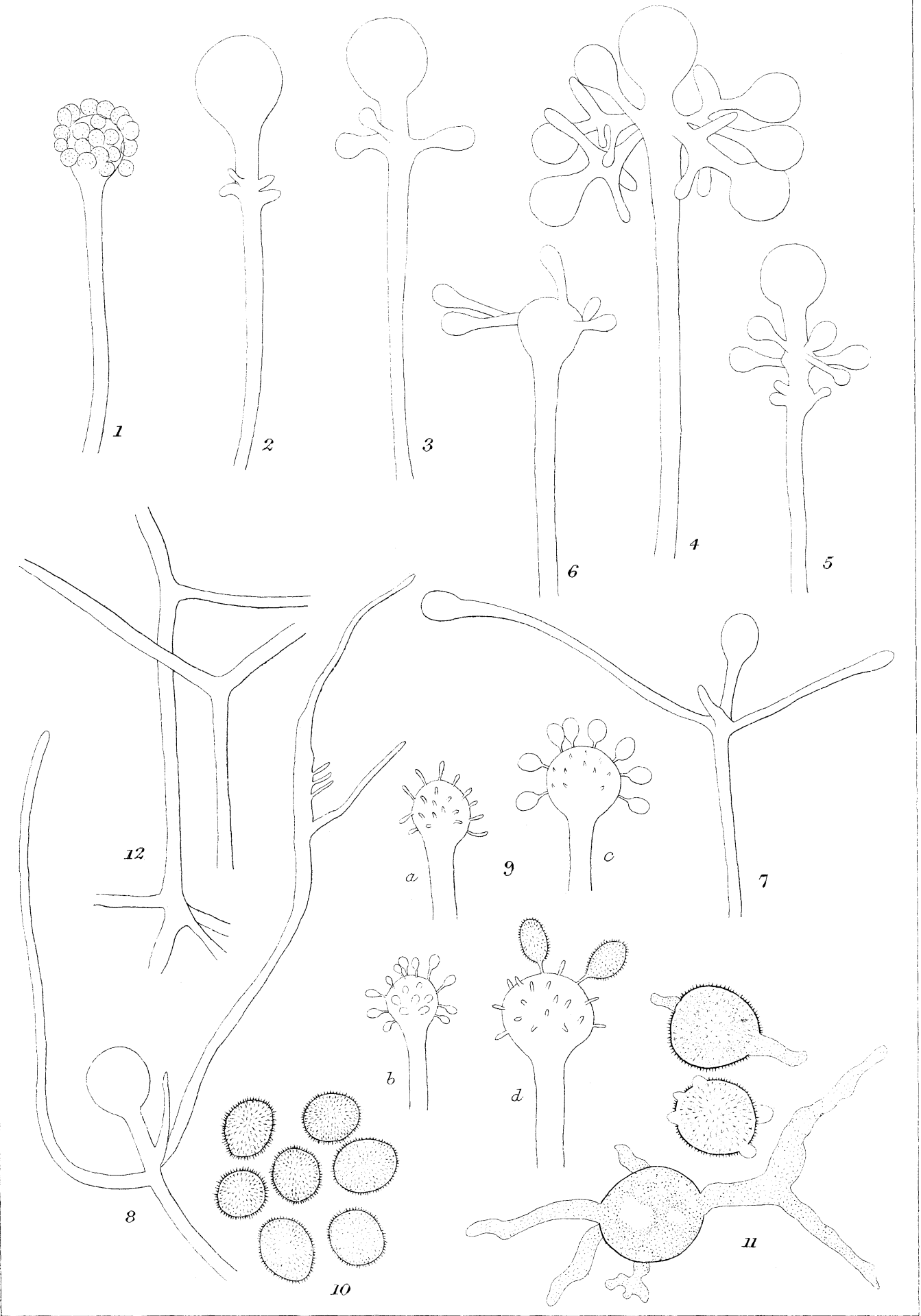


Actinocephalum japonicum nov. gen. et nov. spec.

ron

\title{
K. Saito.
}

\author{
mit 1 Tafel.
}

Gelegentlich einiger zymotechnischer Luftanalysen fand ich im December 1904 die im Nachfolgenden beschriebene Phycomycetenart auf einer Gelatineplatte im Gährkeller einer Sakébrauerei bei der Stadt Kumagaya, Provinz Musashi, Japan. Da ich in der mir zugänglichen Litteratur keine Beschreibung der Art gefunden habe und da sie durch Bildungsweise und Gestalt ihrer ungeschlechtlichen Fortpflanzungsorgane von den bisher bekannten Arten verschieden ist, glaube ich, dass eine Beschreibung derselben von Interesse sein dürfte.

Der Pilz gehört zu den conidienbildenden Mucorineen; seine Conidienträger und daraus ausgebildeten Conidien zeigen zugleich, dass er überhaupt ein neues Genus repräsentiert, das einerseits der Gattung Choetocladium und andererseits den Gattungen Syncephalis und Syncephalastrum am nächsten steht. Ich habe also den Namen Actinocephalum vorschlagen, weil die Anordnung der Nebenköpfe einem Strahlenkranze ähnlich ist, und nannte die Art Actinocephalum japonicum.

Die Vegetation auf dem Reis ist zuerst weiss, bald darauf graulich. Die Substratmycelien sind reich verästelt, anfangs einzellig, im Alter mit vereinzelten Querwänden versehen. Sie sind von variabler Dicke, farblos und glattwandig. Nach einigen Tagen bilden sich vom Mycel zahlreiche Ausläufer; sie krümmen sich verschiedenartig und verbreiten sich nach allen Richtungen auf der Substratfläche (Fig. 12). Aus den Enden der Ausläufer gehen sie zu den Conidienträgern über.

Die Conidienträger entspringen einzeln aus den Mycelien. Sie sind in folgender Weise verzweigt. Die Hauptachse bringt ihr Spitzenwachsthum frühzeitig zum Abschluss durch Bildung einer terminalen kugelig erweiterten Kopflblase. In kleinem Abstande von der Endblase schwillt der Conidienträger ein wenig an, und aus dieser Anschwellung entstehen quirlig drei bis mehrere Aeste, die nicht selten die Höhe des Hauptstammes weit übertreffen. An allen diesen Nebenachsen nehmen dann in derselben Weise 
Achsen zweiter Anordnung ihren Ursprung. Das Ende jeder Nebenachse ist auch kopfig erweitert und alle Kopfe sind mit einer Anzahl dicht gestellter winziger Fortsütze besetzt, die je eine Conidie abschnüren, so dass sie aspergillusartig erscheint (Fig. 1-4, 7, 9).

Ausser dieser terminalen Aestebildung entstehen nicht selten an beliebigen Stellen der Conidienträger wiederum einzelne oder quirlige Verzweigungsaeste (Fig. 5).

Anstatt dass die Spitze des aufstrebenden Hauptstammes der Conidienträger nach und nach die Conidien ausbildet, tritt die Schwellung hier nur als Vorstufe auf. Sie selbst wird nicht zum Kopf, sondern treibt eine oder mehrere kurze oder langfädige Ausstülpungen hervor, welche nun ihrerseits erst mit je einer conidientragenden Blase sich krönen können (Fig. 6).

In einigen Fällen wachsen Hauptstamm und Nebenaeste der Conidienträger nicht in normaler Weise, sondern bildet ein gewöhnliches Mycel, das haufig sehr lang wachsen und eventuell sich verzweigen kann (Fig. 8).

Die Blasen, welche die Hauptzweige abschliessen, sind grösser als dièenigen, welche auf kurzen quirligen Aesten sitzen und die Endblasen wie mit einem Kranze umgeben. Die Hauptblasen sind kugelig, durchschnittlich 25-55 $\mu$ im Durchmesser. Die Nebenblasen erreichen eine Grösse von nicht über $40 \mu$ im Durchmesser.

Die Conidien entstehen auf jedem Fortsatze der Blasen einzeln, sind also durch einmalige Abschnürung entstanden. Sie sind kugelig bis oval, 20 oder $18 \times 21 \mu$ gross, bei der Reife leicht abfallend und ihre Membran ist hyalin und feinwarzig (Fig. 10). Vor der Keimung schwellen die Conidien mehr oder weniger an, wonach ein oder mehrere Keimschläuche ausgesandt werden (Fig. 11).

Unter irgend einer Nährbedingung konnten die Gemmen und Zygosporen nie gebildet werden.

Auf dem Reis wächst der Pilz am üppigsten, mit grauem Conidienrasen. Weniger günstig sind Nährgelatine, -Agar, Kojidecoct u. a. Besondere chemische Wirkungen bringt die Art nicht hervor. Bei $20-25^{\circ}$ entwickelt sie sich sehr rasch und üppig.

Die Gattungen, welche unserer Art nach der vorliegenden Beschreibung am meisten ähnein, sind Chotocladium und Syncephalastrum.

Von der erstgenannten Gattung unterscheidet sich Actinocephalum durch die stets vorkommenden kopfig angeschwollenen Astenden verzweigter Träger, und dadurch, dass die Conidienträger niemals mehrfach sparrigquirlig verästelt sind. 
Von Syncephalastrum unterscheidet er sich durch seine, stets einzeln stehende Conidien, und dadurch, dass die letzteren feinwarzig sind.

Solange die Zygosporen bei unserer Art nicht beobachtet sind, sind wir nicht im Stande die Familie zu bestimmen. Ob Actinocephalum also dem Chcetocladiacece oder Piptocephalidacece angehört, oder man für ihn eine besondere Familie anzusetzen habe, muss dahin gestellt bleiben.

Die Diagnose unseres neuen Phycomycetes ist die folgende:

Actinocephalum japonicum nov. gen. et nov. spec.

Caespitulo griseo, myceleo inaequali, ramoso, citra substratum expanso; hyphis sporangiferis erectis, basi rhizoidibus destitutis, generaliter verticillatis ramosis, ramis capitulato-inflatis, diametro vesiculis $25-55 \mu$; conidiis globosis vel ovalibus, $20 \mu$ vel $18 \times 21 \mu$, monospermis in processibus insertis, hyalinis, echinulatis, zygosporis et chlamydosporis ignotis.

\section{Erklärung der Tafel.}

Fig. 1-4 ( × 250): Entwickelungsstadien der Conidienträger.

Fig. $5(\times 250)$ : Conidienträger mit zwei quirliger Verzweigungen.

Fig. $6(\times 250)$ : Anschwellung der Conidienträger, welche vier fädige Ausstülpungen hervortreibt.

Fig. $7(\times 250)$ : Conidienträger mit langen Seitenzweigen.

Fig. $8(\times 250)$ : Zu normalen Mycelien umgewandelte Seitenzweige der Conidienträger.

Fig. $9(\times 250)$ : Entwickelungsweise der Conidien auf der Blase.

Fig. $10(\times 560)$ : Conidien.

Fig. $11(\times 560)$ : Keimungsstadien der Conidien.

Fig. 12 ( $\times 250)$ : Verzweigung der Luftmycelien. 